Results 410 ultrasounds were performed, $3 \quad(<1 \%)$ of these were in the patient's own home. All scans were performed by a Palliative Medicine Consultant or Clinical Fellow trained in use of FASP.

Clinical indication was broadly split into malignant and non-malignant, $64 \%$ vs.36\% respectively.

Identified indications include; ascites 74\% (302); urinary retention 19\% (78); constipation or overflow 5\% (9); deep vein thrombosis $1 \%$ (5); pleural effusion <1\% (3); assessment of mass $<1 \%$ (2) and spontaneous bacterial peritonitis $<1 \%$ (1). $41 \%$ of the 302 ultrasounds for ascites, resulted in paracentesis. Major and minor complications rates, $0 \%$ vs. $8 \%$ respectively, were reviewed over 4 weeks. Failure of procedure was the highest minor complication rate at $2 \%$. Persistent leakage from drain site, infection, local skin reaction and an asymptomatic drop in blood pressure had complication rates of $<1 \%$ each. Data trends show the number of ultrasounds performed each year is decreasing, however, paracentesis rates remain relatively stable, suggesting more targeted use.

Conclusions Community ultrasounds reduce hospital transfers, admissions and need to access hospital-based radiology services. With appropriately selected patients, the low complication rates, support continued use of community paracentesis. Patient views and preferences on the location of performing ultrasounds and procedures would be valuable. This combined with projected cost savings, has the potential to demonstrate and endorse the importance of these community-based services.

\section{A REVIEW OF PATIENTS REQUIRING LARGE VOLUME PARACENTESIS FOR MALIGNANT ASCITES}

Sadé Hacking, Katie Frew, Irfan Iqbal Khan, Muhammad Tahir Chohan, Avinash Aujayeb. Northumbria HealtCare NHS Foundation Trust

\subsection{6/spcare-2021-PCC.143}

Introduction Malignancy-related ascites (MRA) carries significant morbidity and mortality, and is usually treated by large volume paracentesis (LVP). There are no local guidelines. LVP occurs via Medical Ambulatory Care (MAC). An indwelling peritoneal catheter (IPC) service exists for recurrent ascites. We hypothesised that analysis of patients with MRA requiring LVP would allow protocol development.

Methods A retrospective analysis of patients requiring LVPs for MRA on MAC between Oct 2018 and Dec 19 was performed with local Caldicott approval. Demographics and outcomes were collected. Descriptive statistical methodology was applied with continuous data presented as mean (standard deviation (SD); range) and categorical variables as frequencies or percentages.

Results 27 patients were identified \{Mean age 68.5 yrs (SD \pm 11.85 ; range: $41-86$ ); $55.5 \%$ female $\}$. Rockwood Clinical Frailty Score was recorded in 18: $1=7.4 \%, 2=18.5 \%$, $3=14.8 \%, 5=7.4 \%$. All patients had at least 2 admissions in the preceding 12 months (mean 5.5 (SD $\pm 2.76 ; 2-15)$. Diagnoses were GI (55.5\%), ovarian (25.9\%), unknown primary $(11.1 \%)$, breast $(3.7 \%)$, and neuroendocrine cancers $(3.7 \%)$. $92 \%$ had metastatic disease, 55\% peritoneal metastases. Presenting symptoms were abdominal swelling/bloating/distension $(100 \%)$, abdominal pain $(22 \%)$ and shortness of breath (11\%). $40.7 \%$ of were readmitted within 30 days for drainage. $37 \%$ were referred and had an IPC. Mean time from first LVP to death was 58.18 days (SD $\pm 54.00 ; 7-210$ ). $66 \%$ of patients had a sample sent for analysis. $38.88 \%$ had positive cytology. 5 samples had biochemical analysis: 4 were transudative (all negative cytology); 1 was exudative (positive cytology). No patients developed hypotension with LVP. 1 patient with transudative MRA developed bacterial peritonitis.

Conclusions This is the first study to determine epidemiological local data on the foregoing. There is clear variance in practice. We suggest that all samples should have biochemical analysis (transudates are to be given prophylactic antibiotics) and all patients should be referred for consideration of IPC.

\section{Posters 126 - 138 | Symptom Management}

\section{A SCOPING REVIEW OF THE IMPLEMENTATION OF NON-MEDICAL DEVICES FOR THE SELF-MANAGEMENT OF CHRONIC BREATHLESSNESS}

Aliya Syahreni Prihartadi, Giovanna Impelliziere Licastro, Mark Pearson, Miriam J Johnson, Tim Luckett, Flavia Swan. Wolfson Palliative Care Research Centre, Hull York Medical School, University of Hull, Kingston-Upon-Hull, Faculty of Health, University of Technology Sydney (UTS), Sydney, Australia

\subsection{6/spcare-2021-PCC.144}

Background Non-medical devices such as the handheld fan (fan), mobility aids and inspiratory muscle training (IMT) devices offer important benefits for patient self-management of chronic breathlessness. We examined the published evidence regarding barriers and facilitators to implementation of nonmedical devices for the self-management of chronic breathlessness from the perspectives of patients, carers and clinicians.

Methods MEDLINE, EMBASE, SCOPUS, EBSCO and the Cochrane Database of Systematic Reviews were searched. Papers were imported into EndNote and Rayyan for review against a priori eligibility criteria. These were; i) any study design including randomised controlled trials, observational, interviews, primary and secondary analyses of data, ii) patients; adults with chronic breathlessness, carers', and clinicians, iii) exposure to the fan, mobility aids and IMT devices, iv) and outcome data regarding implementation of these devices for self-management of chronic breathlessness.

Data relevant to use were extracted and categorised as potential implementation barriers and facilitators, and a narrative synthesis exploring reasons for similarities and differences conducted.

Results Seven studies were included; five fan and two mobility aid (wheeled walkers with seats) studies. All of the studies presented patient use of non-medical devices only. The fan was implemented for self-management of chronic breathlessness at home whereas mobility aids were used mainly for outdoor activities. Key barriers to implementation of non-medical devices were: appearance; credibility; self-stigma; technical specifications. Common facilitators were ease of use and feeling safe and secure with the device.

Conclusion Limited evidence exists for the implementation of non-medical devices by patients for the self-management of chronic breathlessness. We highlight the lack of studies on carers, clinicians, IMT devices and the use of applied implementation science theory. Further implementation research is 